\title{
Stochastic Behaviour Analysis of Adaptive Averaging Step-size Sign Normalised Hammerstein Spline Adaptive Filtering
}

Theerayod Wiangtong ${ }^{1}$, Sethakarn Prongnuch ${ }^{*}$, Suchada Sitjongsataporn ${ }^{3}$

${ }^{1}$ Department of Electrical Engineering, Faculty of Engineering, King Mongkut's Institute of Technology Ladkrabang Bangkok, 10520, Thailand

${ }^{2}$ Department of Computer Engineering, Faculty of Industrial Technology, Suan Sunandha Rajabhat University Bangkok, 10300, Thailand

${ }^{3}$ Department of Electronic Engineering, Mahanakorn Institute of Innovation (MII), Faculty of Engineering and Technology Mahanakorn University of Technology, Bangkok, 10530, Thailand

A R T I C L E I N F O

Article history:

Received: 18 November, 2020

Accepted: 09 January, 2021

Online: 28 January, 2021

Keywords:

Hammerstein function

Spline adaptive filter

Sign algorithm

Stochastic gradient descent

\section{A B S T R A C T}

We introduce a sign algorithm based on the normalised least mean square with Hammerstein adaptive filtering using adaptive averaging step-size mechanism, which is derived by the minimised absolute a posteriori squared error. To improve the performance by reducing computational complexity, we suggest an adaptive averaging using energy of errors to update step-size variant. The analysis of convergence behaviour and mean square performance are derived. Experimental results reveal that the proposed algorithm can perform better than the least mean square approach based on the Hammerstein model of adaptive filtering.

\section{Introduction}

Advantages of the adaptive linear filters are simply used in various applications. However, the linear models underperform when comparing with the nonlinear models. Over the last few years, the spline adaptive filter (SAF) structure [1], [2] i.e. Hammerstein spline filtering [3] in cascade architecture [4] has been applied in many practical engineering fields as for example. SAFs based on the set-membership approach [5] and infinite impulse response [6] have been introduced for using in the impulsive noise environment.

In some particular nonlinear problems, the Hammerstein SAF (HSAF) [3], the combined Wiener-Hammerstein SAF [1], and cascade SAFs or sandwich SAF models [4] based on the least mean square (LMS) can operate effectively to identify for nonlinear systems identifications. It provides a good potential for low cost implementation in hardware. Based on the normalised version of LMS (NLMS) on HSAF have been performed in [7]-[10]. In [7], the authors have pointed out the derivation of HSAF on NLMS. In [8], the authors have proposed the performance analysis of HSAF based on the stochastic gradient algorithm. To counteract with impulsive noises, the sign normalised Hammerstein spline adaptive filtering has been proposed in [9] and its performance shown in [10].

In order to reduce the computational requirements of NLMS, the simplifications of NLMS approach in the form of a sign algorithm has been presented in several applications [11]-[16]. To further diminish and protect the impulse noise, the sign normalised SAF [11], [12] and sign subband adaptive filterings with variable step-size parameter [13]- [15] have been designed. In [16], the robust shrinkage normalised version of sign algorithm has been implemented.

Recently, an approach based on the stochastic gradient method for improving the convergence rate based on SAFs has been established in [3], [17]-[19] resulting in the low computation and high performance. For nonlinear systems, the authors in [17] have presented NLMS for SAF. In [18], the adaptive step-size mechanism for SAF has been proposed with the fast convergence and robustness. In [19], the authors have proposed the normalised version of least mean square algorithm for spline adaptive filtering based on the adaptive step-size method with the averaging energy on the previous and present errors and their properties of stability analysis have been introduced.

*Corresponding Author: Sethakarn Prongnuch, Suan Sunandha Rajabhat University, Bangkok, Thailand, sethakarn.pr@ ssru.ac.th 


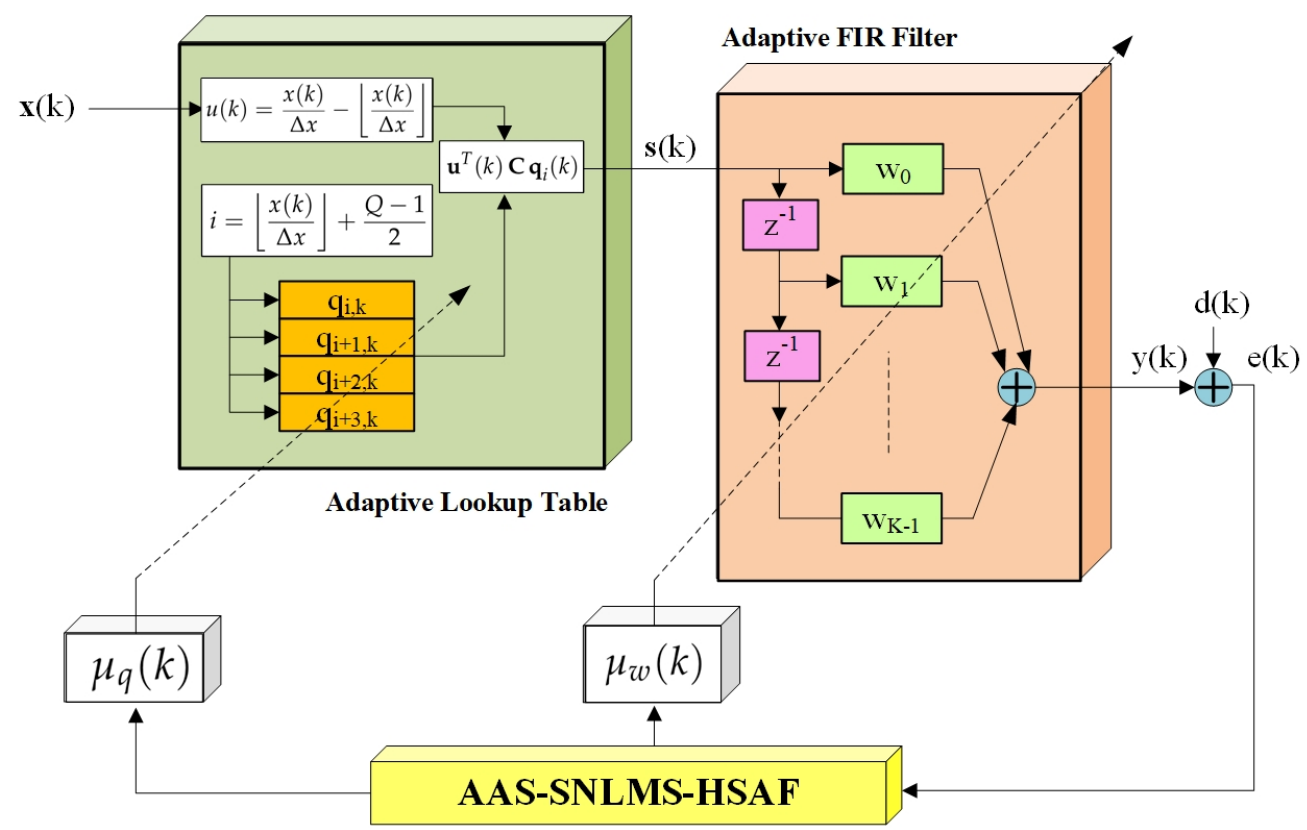

Figure 1: Proposed Adaptive Averaging Step-size Sign Normalised Least Mean Square algorithm for Hammerstein Spline Adaptive Filtering (AAS-SNLMS-HSAF).

In this paper, we propose a statistical behaviour analysis of a Hammerstein spline adaptive filter based on the sign version of normalised least mean square algorithm with adaptive averaging step-size (AAS-SNLMS-HSAF). To enhance the convergence speed with low computational complexity, the adaptive averaging step-size algorithm is suggested.

Also, the performance analysis of sign version of NLMS-HSAF based on adaptive step-size mechanism using averaging energy of errors are derived. We encounter the relationship between the step-size parameter and the mean square error from the analysis that can be examined numerically in the computer simulations for an example of system identification.

This paper is organised as following. Section 2 clarifies the HSAF structure based on LMS approach in brief. Section 3 introduces the constraint criterion of the cost function based on the stochastic gradient descent with the adaptive averaging step-size mechanism. The energy of errors is exploited to update the variant of step-size parameter. Section 4 explains how to derive the convergence analysis of HSAF-based algorithm that consists of the convergence properties and the mean square behaviour analysis. Furthermore, the simulation experiment design and experimental results are detailed in Section 5 and Section 6 . Finally, Section 7 discusses and Section 8 concludes the proposed algorithm.

\section{Hammerstein Spline Adaptive Filtering}

The structure of Hammerstein spline adaptive filtering (HSAF) is depicted in Figure 1 It consists of a nonlinear model controlled by adaptive lookup table (LUT) [20] in which the adaptive control points vector is interpolated by the spline function and adaptive linear finite impulse response (FIR) filter [7].
The vectors $\mathbf{x}_{k}$ and $\mathbf{s}_{k}$ at symbol $k$ appear the input and output vectors of adaptive LUT as

$$
\begin{aligned}
\mathbf{s}_{k} & =\mathbf{u}_{k}^{T} \mathbf{C} \mathbf{q}_{i, k}, \\
\mathbf{u}_{k} & =\left[\begin{array}{llll}
u_{k}^{3} & u_{k}^{2} & u_{k} & 1
\end{array}\right]^{T},
\end{aligned}
$$

where $C$ is a spline matrix, $\mathbf{q}_{i_{k}}$ is the control point vector as $\mathbf{q}_{i, k}=\left[\begin{array}{llll}q_{i, k} & q_{i+1, k} & q_{i+2, k} & q_{i+3, k}\end{array}\right]^{T}$. The local parameter $u_{k}$ and index $i$ can be estimated as [3]

$$
\begin{aligned}
u_{k} & =\frac{x_{k}}{\Delta x}-\left\lfloor\frac{x_{k}}{\Delta x}\right\rfloor, \\
i & =\left\lfloor\frac{x_{k}}{\Delta x}\right\rfloor+\frac{Q-1}{2},
\end{aligned}
$$

where $\Delta x$ is the uniform space between two-adjacent control points, $Q$ is the number of control points, and operator $\lfloor\cdot\rfloor$ is floor operator.

We can obtain the minimised cost function based on LMS for HSAF as

$$
J\left(\mathbf{w}_{k}, \mathbf{q}_{i_{k}}\right)=\min _{\mathbf{w}, \mathbf{q}}\left\{\frac{1}{2} e_{k}^{2}\right\},
$$

where $e_{k}$ is given as [3]

$$
e_{k}=d_{k}-y_{k}=d_{k}-\mathbf{w}_{k-1}^{T} \mathbf{s}_{k},
$$

where $y_{k}$ is the HSAF output, $d_{k}$ is the desired signal and $\mathbf{w}_{k}$ is the adaptive FIR coefficient vector.

By using the chain rule, the cost function in (5) is derivative with respect to (w.r.t.) $\mathbf{w}_{k}, \mathbf{q}_{i, k}$. The result is set to zero to find optimal points. So, the adaptive FIR coefficient vector $\mathbf{w}_{k}$ and the update control points coefficient vector $\mathbf{q}_{i, k}$ are demonstrated in the recursive form as

$$
\begin{aligned}
& \therefore \mathbf{w}_{k+1}=\mathbf{w}_{k}+\mu_{w} \mathbf{s}_{k} e_{k}, \\
& \therefore \mathbf{q}_{i, k+1}=\mathbf{q}_{i, k}+\mu_{q} \mathbf{u}_{k} \mathbf{C}^{T} \mathbf{w}_{k} e_{k},
\end{aligned}
$$

where $\mu_{w}$ and $\mu_{q}$ are the fixed step-size parameters. 


\section{Proposed Adaptive Averaging Step-size Sign Normalised Least Mean Square Algorithm}

We extend the scope of sign algorithm into Hammerstein spline adaptive filtering (HSAF) based on the normalised version of LMS, named SNLMS-HSAF derived by minimised absolute a posteriori error as shown in Figure 1 .

The constraints of this optimisation problem are formulated as

$$
\min _{\mathbf{w}}\left\{e_{k}^{\prime 2}\right\}=\left\{d_{k}-\mathbf{w}_{k+1}^{T} \mathbf{s}_{k}\right\}^{2},
$$

subject to

$$
\left\|\mathbf{w}_{k+1}-\mathbf{w}_{k}\right\|^{2} \leq \xi^{2} \text { and }\left\|\mathbf{q}_{i, k+1}-\mathbf{q}_{i, k}\right\|^{2} \leq \xi^{2},
$$

where $e_{k}^{\prime}$ is a posteriori error and $\xi^{2}$ is a small parameter in the gradual update of coefficient vectors.

From [9], the constrained cost function using Lagrange multiplier can be illustrated by

$$
\mathfrak{J}\left(\mathbf{w}_{k}\right)=\min _{\mathbf{w}}\left\{e_{k}^{\prime 2}\right\}+\frac{\lambda_{0}}{\mathbf{u}_{k}^{T} \mathbf{u}_{k}} \cdot\left[\left\|\mathbf{w}_{k+1}-\mathbf{w}_{k}\right\|^{2} \leq \xi^{2}\right],
$$

where $\lambda_{0}$ is the Lagrange multiplier.

Hence, the derivation of $\mathfrak{J}\left(\mathbf{w}_{k}\right)$ in 111 w.r.t. $\mathbf{w}_{k}$ is set to zero,

$$
-2 \mathbf{s}_{k} \cdot \operatorname{sgn}\left\{e_{k}^{\prime}\right\}+2 \frac{\lambda_{0}}{\mathbf{u}_{k}^{T} \mathbf{u}_{k}} \cdot\left\|\mathbf{w}_{k+1}-\mathbf{w}_{k}\right\|=0
$$
by

Therefore, the adaptive FIR vector $\mathbf{w}_{k}$ is recursively formulated

$$
\mathbf{w}_{k+1}=\mathbf{w}_{k}+\mu_{w_{k}} \frac{\mathbf{s}_{k} \cdot \operatorname{sgn}\left\{e_{k}^{\prime}\right\}}{\mathbf{u}_{k}^{T} \mathbf{u}_{k}+\varepsilon}
$$

where $\mu_{w_{k}}$ is an adaptive step-size of $\mathbf{w}_{k}$ and $\operatorname{sgn}\{\cdot\}$ is the sign function. The regularisation parameter $\varepsilon$ is a small constant.

Similarly, the constraints of the cost function regarding with $\mathbf{q}_{i, k}$ can be shown as

$$
\mathfrak{J}\left(\mathbf{q}_{i, k}\right)=\min _{\mathbf{q}}\left\{e_{k}^{\prime 2}\right\}+\frac{\tilde{\lambda}_{0}}{\mathbf{u}_{k}^{T} \mathbf{u}_{k}} \cdot\left[\left\|\mathbf{q}_{i, k+1}-\mathbf{q}_{i, k}\right\|^{2} \leq \xi^{2}\right] .
$$

Considering the derivation of $\mathfrak{J}\left(\mathbf{q}_{i, k}\right)$ in 13 w.r.t. $\mathbf{q}_{i, k}$ is equal to zero, we have

$$
-2 \mathbf{u}_{k}^{T} \cdot \mathbf{C} \cdot \mathbf{w}_{k} \cdot \operatorname{sgn}\left\{e_{k}^{\prime}\right\}+2 \frac{\tilde{\lambda_{0}}}{\mathbf{u}_{k}^{T} \mathbf{u}_{k}} \cdot\left\|\mathbf{q}_{i, k+1}-\mathbf{q}_{i, k}\right\|=0 .
$$

Finally, the updated control points vector $\mathbf{q}_{i, k}$ is

$$
\mathbf{q}_{i, k+1}=\mathbf{q}_{i, k}+\mu_{q_{k}} \frac{\mathbf{u}_{k}^{T} \cdot \mathbf{C} \cdot \mathbf{w}_{k} \cdot \operatorname{sgn}\left\{e_{k}^{\prime}\right\}}{\mathbf{u}_{k}^{T} \mathbf{u}_{k}+\varepsilon}
$$

where $\mu_{q_{k}}$ is an adaptive step-size of $\mathbf{q}_{i, k}$ and the regularisation parameter $\varepsilon$ is a small constant.

To enhance the performance by reducing computational complexity, we modify an estimation of the energy of errors for updating average step-size parameter from the present and previous errors of systems [21].

Since, the adaptive averaging step-size $\mu_{w_{k}}$ of $\mathbf{w}_{k}$ can be implemented following [19]

$$
\begin{aligned}
\mu_{w_{k}} & =\gamma_{w} \cdot \mu_{w_{k-1}}+\rho_{w} \cdot \zeta_{k}^{2}, \\
\zeta_{k} & =\sigma \cdot \zeta_{k-1}+(1-\sigma) \cdot e_{k}^{\prime 2},
\end{aligned}
$$

where $\rho_{w}$ is a scaling variable, $\sigma$ is close to 1 and $0<\gamma_{w}<1$.

Therefore, the adaptive step-size $\mu_{q_{k}}$ of $\mathbf{q}_{i, k}$ is determined by

$$
\mu_{q_{k}}=\gamma_{q} \cdot \mu_{q_{k-1}}+\rho_{q} \cdot e_{k}^{2}
$$

where $0<\gamma_{q}<1$ and $\rho_{q}>0$.

The summary of the proposed sign normalised version of least mean square algorithm using adaptive averaging step-size approach for HSAF (AAS-SNLMS-HSAF) is presented in Table. 1.

\section{Convergence Analysis}

In this section, we focus on the derivation and analysis of the relationship between the step-size and the mean square error. These approaches are to ensure the optimal convergence at the steady-state condition.

\subsection{Convergence Properties}

According to enhance the performance of SNLMS-HSAF-based algorithm, we examine the optimal learning rate of step-size parameters for adaptive FIR coefficient vector $\mathbf{w}_{k}$ and update control points vector $\mathbf{q}_{i, k}$ as follows.

We determine the a posteriori error $e_{k}^{\prime}$ as

$$
e_{k}^{\prime}=d_{k}-\mathbf{w}_{k+1}^{T} \cdot \mathbf{s}_{k} .
$$

The desired response $d_{k}$ can be decomposed into the input vector $\mathbf{s}_{k}$ and the residual error is defined as

$$
d_{k}=\mathbf{w}_{o p t}^{T} \cdot \mathbf{s}_{k}+e_{k}^{\min },
$$

where $\mathbf{w}_{\text {opt }} \in \mathcal{R}^{N}$ is the optimal Wiener solution vector and $e_{k}^{\min }$ is the residual error assumed as a Gaussian noise with zero mean and finite variance.

Substituting 20] into (19), then we can obtain an expression to estimate error as

$$
e_{k}^{\prime}=e_{k}^{\min }-\Delta \mathbf{w}_{k}^{T} \cdot \mathbf{s}_{k},
$$

where $\Delta \mathbf{w}_{k} \in \mathcal{R}^{N}$ is the difference of the adaptive FIR coefficient vectors $\mathbf{w}_{k}$ defined by

$$
\Delta \mathbf{w}_{k}=\mathbf{w}_{k+1}-\mathbf{w}_{k}=\frac{\mu_{w_{k}} \cdot \mathbf{s}_{k} \cdot \operatorname{sgn}\left\{e_{k}^{\prime}\right\}}{\mathbf{u}_{k}^{T} \mathbf{u}_{k}+\varepsilon} .
$$

Substituting 22, into 21] gives

$$
e_{k}^{\min }-e_{k}^{\prime}=\mu_{w_{k}} \frac{\left(\mathbf{s}_{k}^{T} \cdot \mathbf{s}_{k}\right) \cdot \operatorname{sgn}\left\{e_{k}^{\prime}\right\}}{\mathbf{u}_{k}^{T} \mathbf{u}_{k}+\varepsilon}
$$


By taking the norm of both sides in (23), the adaptive step-size $\mu_{w_{k}}$ of adaptive FIR coefficient vector $\mathbf{w}_{k}$ can be approximated by

$$
\therefore 0<\mu_{w_{k}}<\frac{\mathbf{u}_{k}^{T} \mathbf{u}_{k}}{\mathbf{s}_{k}^{T} \mathbf{s}_{k}+\varepsilon} .
$$

where $\left|e_{k}^{\prime}\right|<\left|e_{k}^{\min }\right|$.

Correspondingly, we investigate the convergence properties of the update control points vector $\mathbf{q}_{i, k}$ using Taylor series expansion of estimated a posteriori error $e_{k}^{\prime}$ as

$$
e_{k+1}^{\prime}=e_{k}^{\prime}+\frac{\partial e_{k}^{\prime}}{\partial \mathbf{q}_{k}} \cdot \Delta \mathbf{q}_{k},
$$

where the difference of update control points vector $\Delta \mathbf{q}_{k}$ is given by

$$
\Delta \mathbf{q}_{k}=\mu_{q_{k}} \frac{\mathbf{u}_{k}^{T} \cdot \mathbf{C} \cdot \mathbf{w}_{k} \cdot \operatorname{sgn}\left\{e_{k}^{\prime}\right\}}{\mathbf{u}_{k}^{T} \mathbf{u}_{k}+\varepsilon} .
$$

Differentiating $e_{k}^{\prime}$ in (19]) w.r.t. $\mathbf{q}_{i, k}$ by the chain rule [19], we can get

$$
\begin{aligned}
\frac{\partial e_{k}^{\prime}}{\partial \mathbf{q}_{k}} & =\frac{\partial}{\partial \mathbf{q}_{k}}\left(d_{k}-\mathbf{w}_{k+1}^{T} \cdot \mathbf{u}_{k} \cdot \mathbf{C}^{T} \cdot \mathbf{q}_{i, k}\right) \\
& =-\mathbf{u}_{k} \cdot \mathbf{C}^{T} \cdot \mathbf{w}_{k+1} .
\end{aligned}
$$

Substituting (26) and 27) into 25] gives

$$
e_{k}^{\prime}-e_{k+1}^{\prime}=\frac{\mu_{q_{k}} \cdot\left(\Psi_{k}^{T} \cdot \Psi_{k}\right) \cdot \operatorname{sgn}\left\{e_{k}^{\prime}\right\}}{\mathbf{u}_{k}^{T} \mathbf{u}_{k}+\varepsilon},
$$

where $\Psi_{k}$ is

$$
\Psi_{k}=\mathbf{u}_{k}^{T} \cdot \mathbf{C} \cdot \mathbf{w}_{k} .
$$

Similarly, we take the norm of both sides (28), the adaptive step-size $\mu_{q_{k}}$ of update control points coefficient vector $\mathbf{q}_{i, k}$ can be evaluated as

$$
\therefore 0<\mu_{q_{k}}<\frac{\mathbf{u}_{k}^{T} \mathbf{u}_{k}}{\Psi_{k}^{T} \Psi_{k}+\varepsilon} \text {. }
$$

where $\left|e_{k}^{\prime}\right|>\left|e_{k+1}^{\prime}\right|$.

\subsection{Mean Square Behaviour Analysis}

In this section, we investigate the convergence analysis in forms of the mean square error performance in the steady-state condition.

Remark 1: We assume that the estimated coefficient error vector $\boldsymbol{V}_{w_{k}}$ is under the independent and identically distributed condition with zero mean and finite variance.

We consider following [4] the estimated coefficient error vector $\mathbf{V}_{w_{k}}$ involved the adaptive FIR vector $\mathbf{w}_{k}$ as

$$
\mathbf{V}_{w_{k+1}}=\mathbf{V}_{w_{k}}-\frac{\mu_{w_{k}} \cdot \mathbf{s}_{k} \cdot \operatorname{sgn}\left\{e_{k}^{\prime}\right\}}{\mathbf{u}_{k}^{T} \mathbf{u}_{k}+\varepsilon} .
$$

Table 1: Proposed adaptive averaging step-size algorithm based on sign normalised least mean square algorithm for HSAF (AAS-SNLMS-HSAF).

$$
\begin{aligned}
& \text { 1: ALGORITHM AAS-SNLMS-HSAF () } \\
& \text { 2: } \quad \mathbf{w}(0)=\delta \cdot\left[\begin{array}{llll}
1 & 0 & \ldots & 0
\end{array}\right]^{T}, \quad \mathbf{u}_{k}=\left[\begin{array}{llll}
u_{k}^{3} & u_{k}^{2} & u_{k} & 1
\end{array}\right]^{T} \\
& \mathbf{x}_{k}=\left[\begin{array}{llll}
x_{k} & x_{k-1} & \ldots & x_{k-K+1}
\end{array}\right] \\
& \mathbf{q}_{i, k}=\left[q_{i, k} q_{i+1, k} q_{i+2, k} q_{i+3, k}\right] \\
& \text { 3: } \quad \text { FOR } k=1 \text { TO } K-1 \text {. } \\
& \text { 4: } \quad \mathbf{s}_{k}=\mathbf{u}_{k}^{T} \mathbf{C} \mathbf{q}_{i, k} \\
& \text { 5: } \quad u_{k}=\frac{s_{k}}{\Delta x}-\left\lfloor\frac{s_{k}}{\Delta x}\right\rfloor \\
& \text { 6: } \quad i=\left\lfloor\frac{s_{k}}{\Delta x}\right\rfloor+\frac{Q-1}{2} \\
& \text { 7: } \quad e_{k}^{\prime}=d_{k}-y_{k}=d_{k}-\mathbf{w}_{k+1}^{T} \mathbf{s}_{k} \\
& \text { 8: } \quad \mu_{w_{k}}=\gamma_{w} \cdot \mu_{w_{k-1}}+\rho_{w} \cdot \zeta_{k}^{2} \\
& \text { 9: } \quad \zeta_{k}=\sigma \cdot \zeta_{k-1}+(1-\sigma) \cdot e_{k}^{\prime 2} \\
& \text { 10: } \mu_{q_{k}}=\gamma_{q} \cdot \mu_{q_{k-1}}+\rho_{q} \cdot e_{k}^{\prime 2} \\
& \text { 11: } \quad \mathbf{w}_{k+1}=\mathbf{w}_{k}+\mu_{w_{k}} \frac{\mathbf{s}_{k} \cdot \operatorname{sgn}\left\{e_{k}^{\prime}\right\}}{\mathbf{u}_{k}^{T} \mathbf{u}_{k}+\varepsilon} \\
& \text { 12: } \quad \mathbf{q}_{i, k+1}=\mathbf{q}_{i, k}+\mu_{q_{k}} \frac{\mathbf{u}_{k}^{T} \cdot \mathbf{C} \cdot \mathbf{w}_{k} \cdot \operatorname{sgn}\left\{e_{k}^{\prime}\right\}}{\mathbf{u}_{k}^{T} \mathbf{u}_{k}+\varepsilon} \\
& \text { 13: } \quad \text { NEXT } k \\
& \text { 14: END ALGORITHM }
\end{aligned}
$$

We can also determine the squared coefficient error vector as

$$
\left\|\mathbf{V}_{w_{k+1}}\right\|^{2}=\left\|\mathbf{V}_{w_{k}}\right\|^{2}-\frac{2 \mathbf{V}_{w_{k}} \mu_{w_{k}} \cdot \mathbf{s}_{k} \cdot \operatorname{sgn}\left\{e_{k}^{\prime}\right\}}{\mathbf{u}_{k}^{T} \mathbf{u}_{k}+\varepsilon}+\left\|\frac{\mu_{w_{k}} \cdot \mathbf{s}_{k} \cdot \operatorname{sgn}\left\{e_{k}^{\prime}\right\}}{\mathbf{u}_{k}^{T} \mathbf{u}_{k}+\varepsilon}\right\|^{2} .
$$

Remark 2: We consider the condition, that

$$
E\left\{\left\|\mathbf{V}_{w_{k+1}}\right\|^{2}\right\} \approx E\left\{\left\|\mathbf{V}_{w_{k}}\right\|^{2}\right\}, k \rightarrow \infty .
$$

By using Remark 2, we can rewrite (32) as

$$
\begin{aligned}
2 \mathbf{V}_{w_{k}}^{T} \cdot \mathbf{s}_{k} \cdot \operatorname{sgn}\left\{e_{k}^{\prime}\right\} & =\frac{\mu_{w_{k}} \cdot\left\|\mathbf{s}_{k}\right\|^{2} \cdot\left|e_{k}^{\prime}\right|^{2}}{\mathbf{u}_{k}^{T} \mathbf{u}_{k}+\varepsilon} \\
2 \epsilon_{w_{k}} \cdot \operatorname{sgn}\left\{e_{k}^{\prime}\right\} & =\frac{\mu_{w_{k}} \cdot\left\|\mathbf{s}_{k}\right\|^{2} \cdot\left|e_{k}^{\prime}\right|^{2}}{\mathbf{u}_{k}^{T} \mathbf{u}_{k}+\varepsilon},
\end{aligned}
$$

where $\epsilon_{w_{k}}$ is denoted by

$$
\epsilon_{w_{k}} \approx \mathbf{V}_{w_{k}}^{T} \cdot \mathbf{s}_{k} .
$$

We proceed the a posteriori error involved with the adaptive FIR vector $\mathbf{w}_{k}$ as

$$
e_{k}^{\prime}=\epsilon_{w_{k}}+\mathbf{V}_{w_{k}} .
$$

By taking the expectation on the left-side of (33) and using 35 at the steady state condition, we can see that

$$
E\left\{\epsilon_{w_{k}} \cdot \operatorname{sgn}\left\{e_{k}^{\prime}\right\}\right\}=E\left\{\epsilon_{w_{k}} \cdot \operatorname{sgn}\left\{\epsilon_{w_{k}}+\mathbf{V}_{w_{k}}\right\}\right\} \approx E\left\{\epsilon_{w_{k}}^{2}\right\} .
$$


The expectation of the squared a posteriori error is then

$$
\begin{aligned}
E\left\{\left(e_{k}^{\prime}\right)^{2}\right\} & \simeq E\left\{\left(\epsilon_{w_{k}}+\mathbf{V}_{w_{k}}\right)^{2}\right\} \\
& =E\left\{\epsilon_{w_{k}}^{2}+\zeta_{w_{k}}^{2}\right\}
\end{aligned}
$$

where $\zeta_{w_{k}}^{2}$ is given by

$$
\zeta_{w_{k}}^{2}=2 \epsilon_{w_{k}} \cdot \mathbf{V}_{w_{k}}+\mathbf{V}_{w_{k}}^{2}
$$

Substituting (36) and 37) into (33), we then obtain

$$
\begin{gathered}
2 E\left\{\epsilon_{w_{k}}^{2}\right\}=\frac{\mu_{w_{k}} \cdot\left\|\mathbf{s}_{k}\right\|^{2} \cdot E\left\{\epsilon_{w_{k}}^{2}+\zeta_{w_{k}}^{2}\right\}}{\mathbf{u}_{k}^{T} \mathbf{u}_{k}+\varepsilon} \\
E\left\{\epsilon_{w_{k}}^{2}\right\}=\frac{\mu_{w_{k}} \cdot\left\|\mathbf{s}_{k}\right\|^{2} \cdot E\left\{\zeta_{w_{k}}^{2}\right\}}{2 \mathbf{u}_{k}^{T} \mathbf{u}_{k}+\varepsilon-\mu_{w_{k}} \cdot\left\|\mathbf{s}_{k}\right\|^{2}}
\end{gathered}
$$

where $\mu_{w_{k}} \ll 1$ is assumed.

Therefore, the excess mean square error (MSE) $\xi_{e x}^{w}$ involved with the adaptive FIR vector $\mathbf{w}_{k}$ can be given by

$$
\therefore \xi_{e x}^{w}=E\left\{\epsilon_{w_{k}}^{2}\right\}=\frac{\mu_{w_{k}} \cdot\left\|\mathbf{s}_{k}\right\|^{2} \cdot E\left\{\zeta_{w_{k}}^{2}\right\}}{2 \mathbf{u}_{k}^{T} \mathbf{u}_{k}+\varepsilon} .
$$

Please note that (40), an approximation of (39), is valid only for small step-size parameter.

In a similar way, we consider the estimated coefficient error vector $\mathbf{V}_{q_{k}}$ concerned with the update control points vector $\mathbf{q}_{i, k}$ as

$$
\mathbf{V}_{q_{k}}=\mathbf{q}_{i, k}-\mathbf{q}_{\text {opt }}
$$

where $\mathbf{q}_{\text {opt }} \in \mathcal{R}^{N}$ is the optimal control points vector and $\mathbf{V}_{q_{k}} \in \mathcal{R}^{N}$.

We can then rewrite the update control points vector $\mathbf{q}_{i, k}$ in (15) by using the coefficient error vector $\mathbf{V}_{q_{k}}$ as follows.

$$
\mathbf{V}_{q, k+1}=\mathbf{V}_{q, k}-\frac{\mu_{q_{k}} \cdot \mathbf{u}_{k}^{T} \cdot \mathbf{C} \cdot \mathbf{w}_{k} \cdot \operatorname{sgn}\left\{e_{k}^{\prime}\right\}}{\mathbf{u}_{k}^{T} \mathbf{u}_{k}+\varepsilon}
$$

The square of coefficient error vector in 42 is computed as

$$
\begin{aligned}
\left\|\mathbf{V}_{q, k+1}\right\|^{2}=\left\|\mathbf{V}_{q, k}\right\|^{2}- & \frac{2 \cdot \mathbf{V}_{q, k} \cdot \mu_{q_{k}} \cdot \mathbf{u}_{k}^{T} \cdot \mathbf{C} \cdot \mathbf{w}_{k} \cdot \operatorname{sgn}\left\{e_{k}^{\prime}\right\}}{\mathbf{u}_{k}^{T} \mathbf{u}_{k}+\varepsilon} \\
& +\left\|\frac{\mu_{q_{k}} \cdot \mathbf{u}_{k}^{T} \cdot \mathbf{C} \cdot \mathbf{w}_{k} \cdot \operatorname{sgn}\left\{e_{k}^{\prime}\right\}}{\mathbf{u}_{k}^{T} \mathbf{u}_{k}+\varepsilon}\right\|^{2} .
\end{aligned}
$$

Remark 3: We assume the condition, that is of

$$
E\left\|\mathbf{V}_{q_{k+1}}\right\|^{2} \approx E\left\|\mathbf{V}_{q_{k}}\right\|^{2}, k \rightarrow \infty
$$

We can get and rewrite (43) using Remark 1 as

$$
2 \cdot \epsilon_{q_{k}} \cdot \operatorname{sgn}\left\{e_{k}^{\prime}\right\}=\frac{\mu_{q_{k}} \cdot\left\|\Psi_{k}\right\|^{2} \cdot\left|e_{k}^{\prime}\right|^{2}}{\mathbf{u}_{k}^{T} \mathbf{u}_{k}+\varepsilon},
$$

where $\epsilon_{q_{k}}$ is given by

$$
\epsilon_{q_{k}} \approx \mathbf{V}_{q_{k}}^{T} \cdot \Psi_{k}
$$

where $\Psi_{k}$ is defined in (29).

We calculate the a posteriori error with the updated control points vector $\mathbf{q}_{i, k}$ as

$$
e_{k}^{\prime}=\epsilon_{q_{k}}+\mathbf{V}_{q_{k}} \text {. }
$$

After taking the expectation on the left-side of (44) and using (46), we get

$$
\begin{aligned}
E\left\{\epsilon_{q_{k}} \cdot \operatorname{sgn}\left\{e_{k}^{\prime}\right\}\right\} & =E\left\{\epsilon_{q_{k}} \cdot \operatorname{sgn}\left\{\epsilon_{q_{k}}+\mathbf{V}_{q_{k}}\right\}\right\} \\
& \approx E\left\{\epsilon_{q_{k}}^{2}\right\} .
\end{aligned}
$$

We determine the square a posteriori error and take the expectation as

$$
\begin{aligned}
E\left\{e_{k}^{\prime}\right\} & \simeq E\left\{\left(\epsilon_{q_{k}}+\mathbf{V}_{q_{k}}\right)^{2}\right\} \\
& =E\left\{\epsilon_{q_{k}}^{2}\right\}+E\left\{\zeta_{q_{k}}^{2}\right\},
\end{aligned}
$$

where $\zeta_{q_{k}}^{2}$ is given by

$$
\zeta_{q_{k}}^{2}=2 \epsilon_{q_{k}} \cdot \mathbf{V}_{q_{k}}+\mathbf{V}_{q_{k}}^{2}
$$

Substituting (47) and 48) into 44) gives us

$$
\begin{aligned}
2 \cdot E\left\{\epsilon_{q_{k}}^{2}\right\} & =\frac{\mu_{q_{k}} \cdot\left\|\Psi_{k}\right\|^{2} \cdot\left[E\left\{\epsilon_{q_{k}}^{2}\right\}+E\left\{\zeta_{q_{k}}^{2}\right\}\right]}{\mathbf{u}_{k}^{T} \mathbf{u}_{k}+\varepsilon} \\
E\left\{\epsilon_{q_{k}}^{2}\right\} & =\frac{\mu_{q_{k}} \cdot\left\|\Psi_{k}\right\|^{2} \cdot\left[E\left\{\zeta_{q_{k}}^{2}\right\}\right]}{\left(2 \mathbf{u}_{k}^{T} \mathbf{u}_{k}+\varepsilon\right)-\mu_{q_{k}} \cdot\left\|\Psi_{k}\right\|^{2}},
\end{aligned}
$$

where $\mu_{q_{k}} \ll 1$ is assumed.

Therefore, the excess MSE $\xi_{e x}^{q}$ with the update control points coefficient vector $\mathbf{q}_{i, k}$ can be expressed by

$$
\therefore \xi_{e x}^{q} \simeq E\left\{\epsilon_{q_{k}}^{2}\right\}=\frac{\mu_{q_{k}} \cdot\left\|\Psi_{k}\right\|^{2} \cdot\left[E\left\{\zeta_{q_{k}}^{2}\right\}\right]}{2 \mathbf{u}_{k}^{T} \mathbf{u}_{k}+\varepsilon} .
$$

Notice that 51) is an approximation of (50) is only applicable for small values of the step-size parameter.

\section{Simulation Experiment Design}

In this section, the experiments are conducted with the system identification through computer simulations to verify the proposed nonlinear adaptive filtering. Flow chart of the proposed AAS-SNLMS for HSAF is shown in Figure 2

The input color signal $x_{k}$ composes of 10,000 samples by averaging over 100 Monte Carlo trials can be generated by the following equation as [3]

$$
x_{k}=\omega \cdot x_{k-1}+\sqrt{1-\omega^{2}} \varphi,
$$

where $\varphi$ is a unitary variance of zero mean white Gaussian noise and $\omega$ is a parameter to determine the correlation level between adjacent samples [2], where $0 \leq \omega<1$.

In these experiments, we set $\omega=0.15,0.75$ and signal to noise ratio (SNR) is of $20,25,30 \mathrm{~dB}$. As shown in [3], simulation parameters are comprised of

$$
\mathbf{w}_{0}=[0.6,-0.4,0.25,-0.15,0.1,-0.05,0.001] .
$$


The target function of nonlinear memoryless determined by a 23-point LUT of update control points vector $\mathbf{q}_{0}$ is interpolated by a uniform third degree spline function using an interval sampling $\Delta x=0.2[3$

$$
\begin{gathered}
\mathbf{q}_{0}=\{-2.20,-2.00,-1.80,-1.60,-1.40,-1.20,-1.00,-0.80, \\
-0.91,-0.40,-0.20,0.05,0.0,-0.40,0.58,1.00,1.00, \\
1.20,1.40,1.60,1.80,2.00,2.20\}
\end{gathered}
$$

The constant matrix $\mathbf{C}$ is called a Catmul-Rom spline matrix as given by [5]

$$
\mathbf{C}=\frac{1}{2}\left[\begin{array}{rrrr}
-1 & 3 & -3 & 1 \\
2 & -5 & 4 & -1 \\
-1 & 0 & 1 & 0 \\
0 & 2 & 0 & 0
\end{array}\right]
$$

For HSAF models, the initial parameters are in the following terms: $\delta=0.001$, number of tap length $M=7$, number of control points $Q=23, \mu_{w}=1.15 \times 10^{-3}, 4.25 \times 10^{-3}$, and $\mu_{q}=1.15 \times 10^{-3}, 2.55 \times 10^{-3}$.

Other initial parameters of the proposed adaptive averaging step-size sign normalised least mean square algorithm based on the Hammerstein spline adaptive filtering (AAS-SNLMS-HSAF) consists of $\mu_{w}(0)=7.5 \times 10^{-4}, 7.5 \times 10^{-3}, 7.5 \times 10^{-2}$ and $\mu_{q}(0)=$ $5.5 \times 10^{-4}, 5.5 \times 10^{-3}, 5.5 \times 10^{-2}$. And the fixed parameter for adaptive averaging step-size approach of the adaptive FIR vector $\mathbf{w}_{k}$ and the update control points $\mathbf{q}_{i, k}$ are set as: $\gamma_{w}=0.97, \gamma_{q}=0.975$, $\sigma=0.975, \rho_{w}=2.75 \times 10^{-3}, \rho_{q}=2.95 \times 10^{-3}$, and $\varepsilon=1 \times 10^{-6}$.

\section{Experimental Results}

The first experiment is carried out to show the effectiveness of the proposed AAS-SLMS algorithm for HSAF performs against the white Gaussian noise. In particular, the mean square error (MSE) of HSAF-based algorithm with the different of $\omega=0.15,0.75$ with $S N R=25,30 \mathrm{~dB}$ presented in Figures 3 and 4 that manifests the proposed AAS-SNLMS-HSAF and the conventional least mean square algorithm based on Hammerstein spline adaptive filtering (LMS-HSAF) [3] with the two different choices of $\omega$ given in [52]. It shows that the MSE directions of the proposed AAS-SNLMSHSAF algorithm can achieve faster convergence rate compared with to the LMS-HSAF algorithm at the steady state condition.

In addition, Figure 5 shows the trajectories of $\mu_{w_{k}}$ of tapweight vector $\mathbf{w}_{k}$ at different initial value of step-size as $\mu_{w}(0)=$ $1.75 \times 10^{-2}, 1.75 \times 10^{-3}, 1.75 \times 10^{-4}$ at $\omega=0.75$ with $S N R=20 \mathrm{~dB}$. Figure 6 conducts the step-size curves $\mu_{q_{k}}$ of control point $\mathbf{q}_{i, k}$ at different initial step-size $\mu_{q}(0)=1.55 \times 10^{-2}, 1.55 \times 10^{-3}, 1.55 \times 10^{-4}$ at the same environment.

Figure 7 depicts the trajectories of $\mu_{w_{k}}$ of tap-weight vector $\mathbf{w}_{k}$ at different initial value of step-size as $\mu_{w}(0)=7.5 \times 10^{-2}, 7.5 \times$ $10^{-3}, 7.5 \times 10^{-4}$ at $\omega=0.75$ with $S N R=25 \mathrm{~dB}$. Figure 8 shows the step-size curves $\mu_{q_{k}}$ of control point $\mathbf{q}_{i, k}$ at different initial step-size $\mu_{q}(0)=5.5 \times 10^{-2}, 5.5 \times 10^{-3}, 5.5 \times 10^{-4}$ at the same environment.

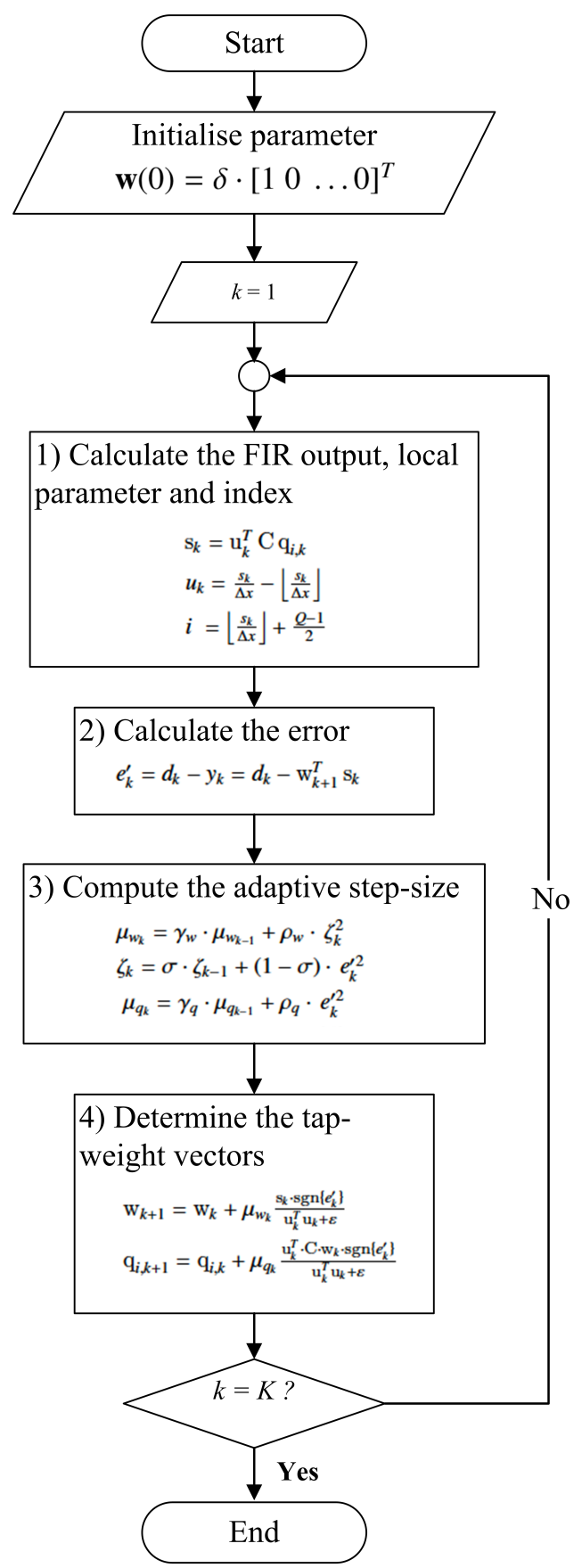

Figure 2: Flow chart of proposed AAS-SNLMS-HSAF algorithm

Figure 9 presents the trajectories of $\mu_{w_{k}}$ of tap-weight vector $\mathbf{w}_{k}$ at different initial value of step-size as $\mu_{w}(0)=1.75 \times 10^{-2}, 1.75 \times$ $10^{-3}, 1.75 \times 10^{-4}$ at $\omega=0.75$ with $S N R=30 \mathrm{~dB}$.

Figure 10 shows the step-size curves $\mu_{q_{k}}$ of control point $\mathbf{q}_{i, k}$ at different initial step-size $\mu_{q}(0)=1.55 \times 10^{-2}, 1.55 \times 10^{-3}, 1.55 \times 10^{-4}$ in the same environment.

Considering the learning rate of the proposed step-size mechanism in terms of both the adaptive step-size parameter $\mu_{w_{k}}$ of $\mathbf{w}_{k}$ and the adaptive step-size $\mu_{q_{k}}$ of $\mathbf{q}_{i, k}$ with different SNR values, they can quickly converge to steady-state conditions compared with the fixed step-size. 


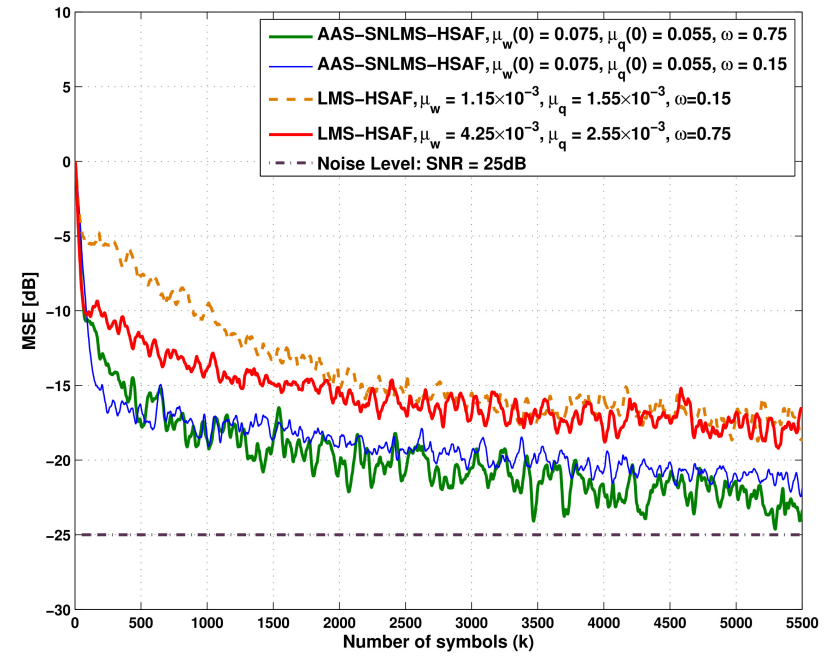

Figure 3: MSE of proposed AAS-SNLMS-HSAF with the different initial stepsize of $\mu_{w}(0), \mu_{q}(0)$ and LMS-HSAF [3] with the fixed step-size $\mu_{w}$ and $\mu_{q}$, when $\omega=0.15,0.75$ and $\mathrm{SNR}=25 \mathrm{~dB}$.

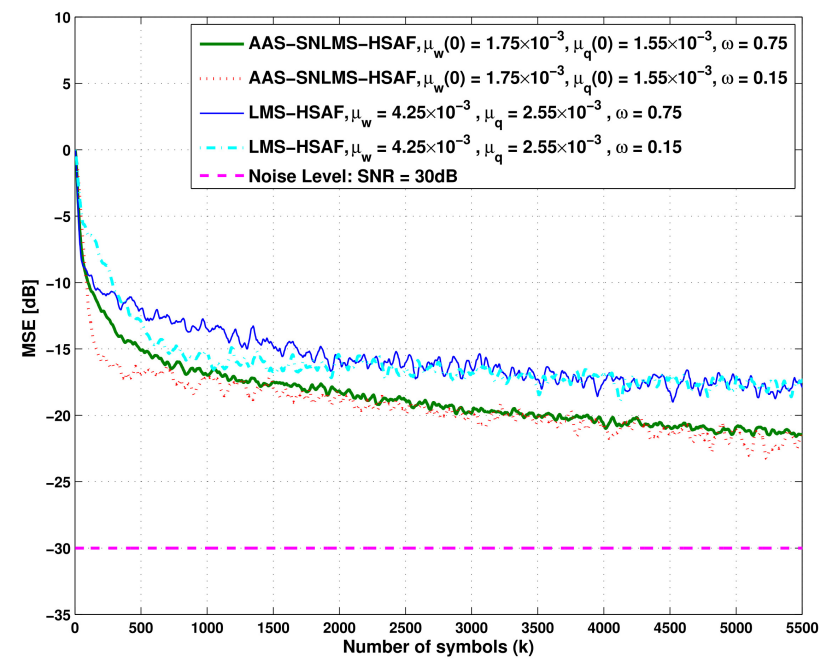

Figure 4: MSE of proposed AAS-SNLMS-HSAF with the different initial stepsize of $\mu_{w}(0), \mu_{q}(0)$ and LMS-HSAF [3] with the fixed step-size $\mu_{w}$ and $\mu_{q}$, when $\omega=0.15,0.75$ and $\mathrm{SNR}=30 \mathrm{~dB}$.

In the second experiment, the electrocardiogram (ECG) [22] from the MIT-BIH Atrial Fibrillation Database [23], [24] is used as a real biomedical input signal, shown in Figure 11 ECG is sampled at $250 \mathrm{~Hz}$. Figure 12 shows the proposed AAS-SNLMS-HSAF and LMS-HSAF [3] using the ECG dataset with the different $\omega$ can achieve better convergence rate compared with the LMS-HSAF algorithm, even the ECG input signal is small.

Figure 13 demonstrates the trajectories of $\mu_{w_{k}}$ of coefficient vector $\mathbf{w}_{k}$ at different $\omega=0.15,0.75$ and the initial value of step-size $\mu_{w}(0)=1.75 \times 10^{-3}$ and $\mu_{q}(0)=1.55 \times 10^{-3}$ with $S N R=25 \mathrm{~dB}$ using the ECG dataset at [24]. Figure 14]presents the the learning curves of $\mu_{q_{k}}$ of control point $\mathbf{q}_{i, k}$ at the same environment. It reveals

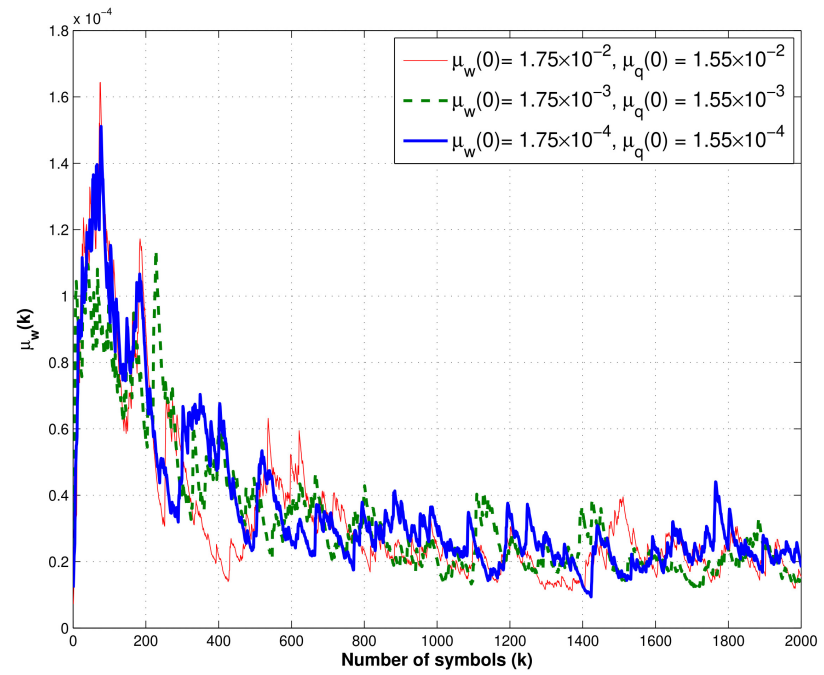

Figure 5: Trajectories of step-size $\mu_{w_{k}}$ of coefficient vector $\mathbf{w}_{k}$ with the different initial step-size parameters $\mu_{w}(0)$, when $\omega=0.75$ and SNR $=20 \mathrm{~dB}$.

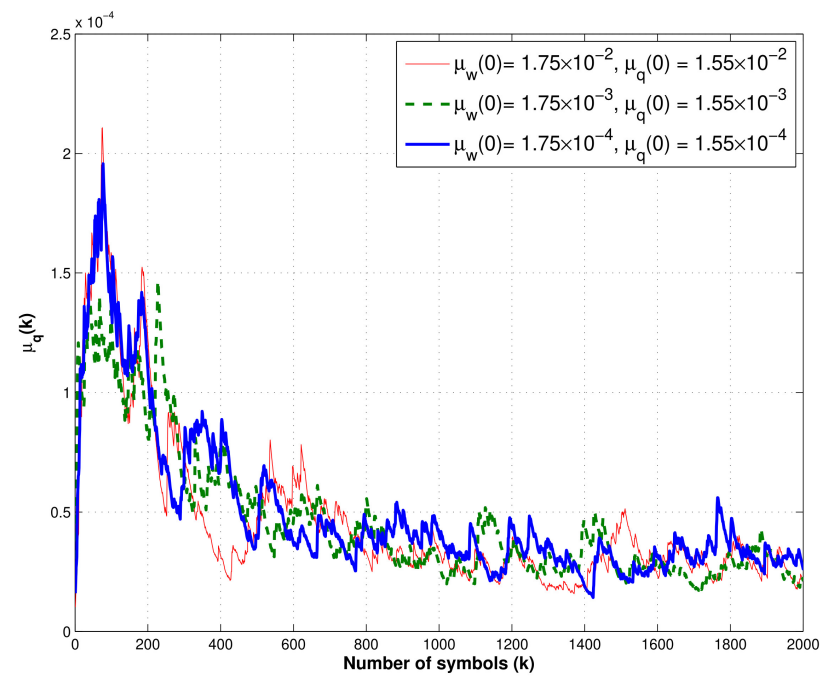

Figure 6: Trajectories of step-size $\mu_{q_{k}}$ of control points coefficient vector $\mathbf{q}_{i, k}$ with the different initial step-size $\mu_{q}(0)$, when $\omega=0.75$ and SNR $=20 \mathrm{~dB}$.

that the proposed adaptive step-size algorithms for both $\mu_{w_{k}}$ and $\mu_{q_{k}}$ can converge to equilibrium points using the real ECG dataset.

\section{Discussion}

The comparison over 100 Monte Carlo trials shows the robustness and superiority of the proposed AAS-SNLMS algorithm over the conventional LMS algorithm for HSAF model. The learning curves of adaptive step-size $\mu_{w_{k}}$ and $\mu_{g_{k}}$ of the proposed AAS-SNLMS algorithm after 10,000 iterations can expedite the convergence rate even the initial values are varied. 


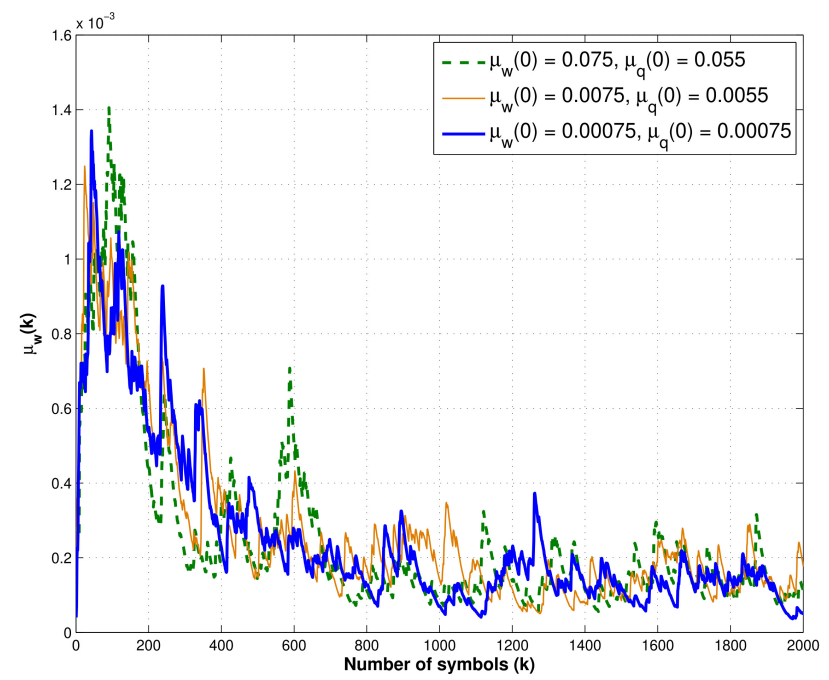

Figure 7: Trajectories of step-size $\mu_{w_{k}}$ of coefficient vector $\mathbf{w}_{k}$ with the different initial step-size $\mu_{w}(0)$, when $\omega=0.75$ and SNR $=25 \mathrm{~dB}$.

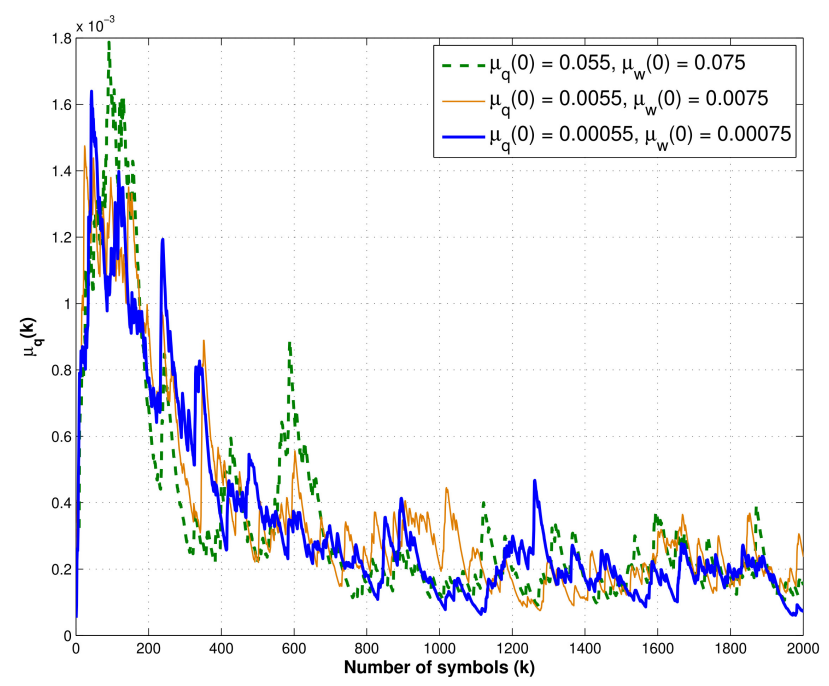

Figure 8: Trajectories of step-size $\mu_{q_{k}}$ of control points coefficient vector $\mathbf{q}_{i, k}$ with the different initial step-size $\mu_{q}(0)$, when $\omega=0.75$ and $\mathrm{SNR}=25 \mathrm{~dB}$.

\section{Conclusion}

This paper presents a sign algorithm based on the normalised least mean square with Hammerstein adaptive filtering by applying an adaptive averaging step-size scheme. The proposed algorithm is developed using the minimised absolute a posteriori squared error. We modify an adaptive averaging step-size mechanism by using the energy of the estimated a posteriori error to update the step-size variant.

Furthermore, we derive the behaviour and mean square performance analysis of a sign algorithm based on the normalised version of least mean square algorithm for Hammerstein spline adaptive

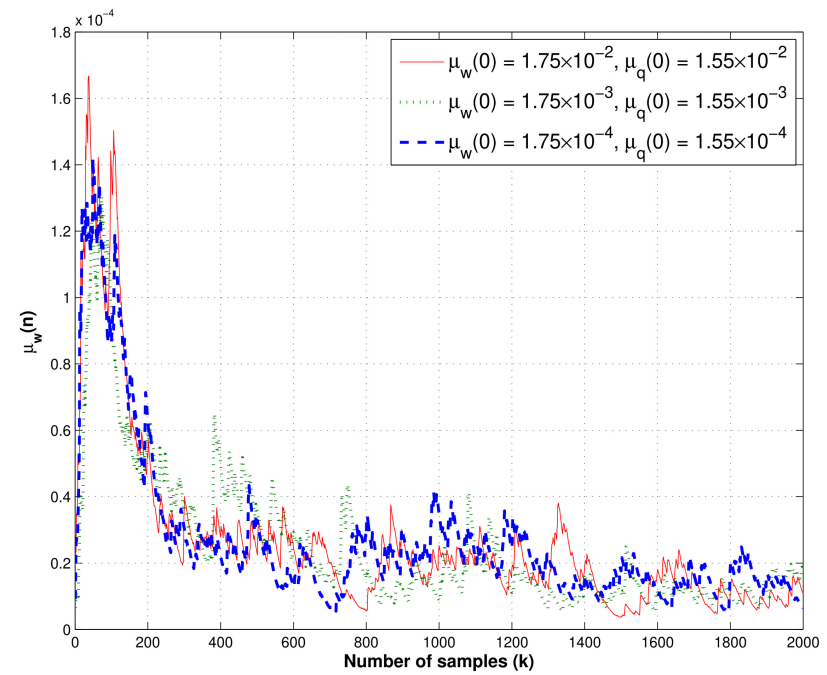

Figure 9: Trajectories of step-size $\mu_{w_{k}}$ of coefficient vector $\mathbf{w}_{k}$ with the different initial step-size $\mu_{w}(0)$, when $\omega=0.75$ and SNR $=30 \mathrm{~dB}$.

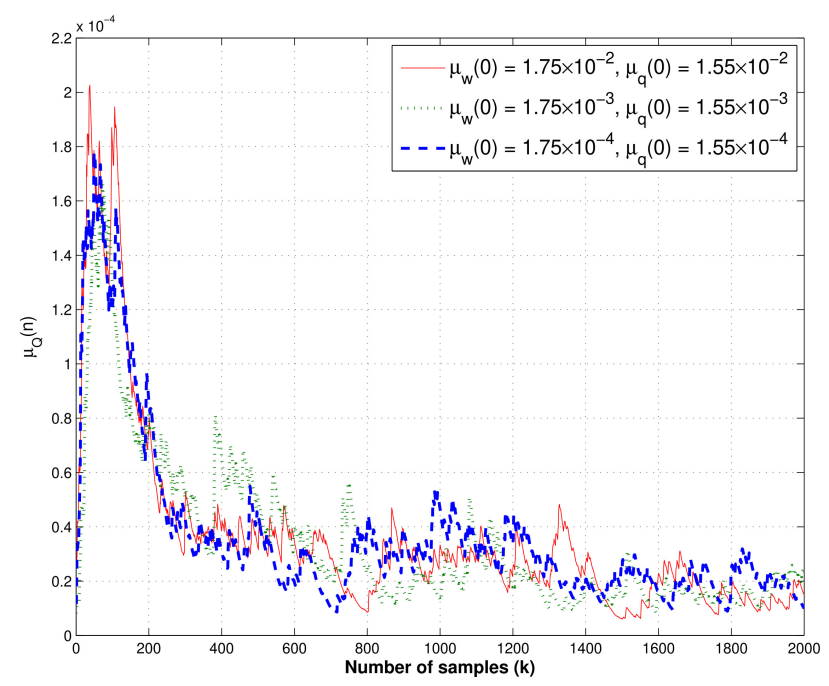

Figure 10: Trajectories of step-size $\mu_{q_{k}}$ of control points coefficient vector $\mathbf{q}_{i, k}$ with the different initial step-size $\mu_{q}(0)$, when $\omega=0.75$ and $\mathrm{SNR}=30 \mathrm{~dB}$.

filtering with the adaptive averaging step-size algorithm. That leads to discover the relationship between the step-size parameter and the mean square error from the analysis. Experimental results clearly show that the proposed algorithm outperforms the conventional least mean square based on the Hammerstein adaptive filter approach.

Hammerstein models are being particularly interested in fields of engineering such as adaptive signal processing, biomedical engineering and data analysis in the nonlinear processes.

Conflict of Interest The authors declare no conflict of interest. 


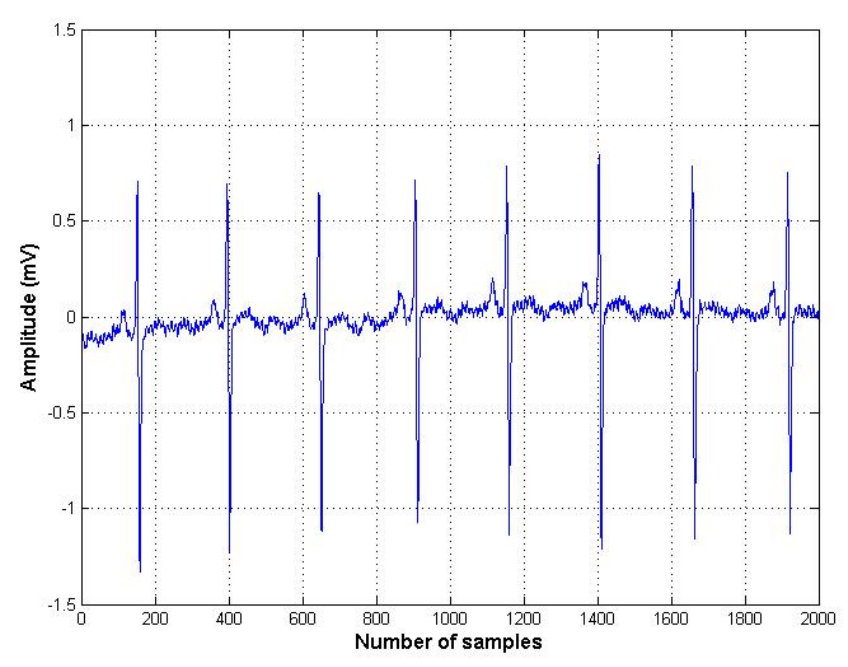

Figure 11: Electrocardiogram (ECG) input signal from MIT-BIH Atrial Fibrillation Database at [24].

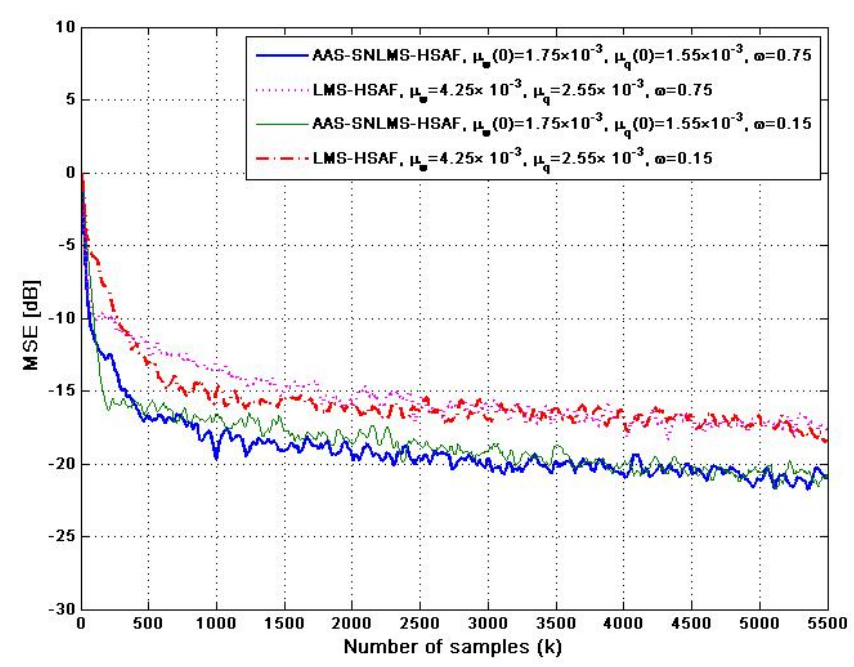

Figure 12: MSE of proposed AAS-SNLMS-HSAF and LMS-HSAF [3] using ECG input signal, when $\omega=0.15,0.75$ and $\mathrm{SNR}=25 \mathrm{~dB}$.

\section{References}

[1] M. Scarpiniti, D. Comminiello and A. Uncini, "Convex Combination of Spline Adaptive Filters", in 2019 IEEE European Signal Processing Conference (EUSIPCO), 1-5, 2019, doi:10.23919/EUSIPCO.2019.8903134.

[2] M. Scarpiniti, D. Comminiello, R. Parisi and A. Uncini, "Nonlinear spline adaptive filtering," Signal Processing, 93(4), 772-783, 2013, doi:10.1016/j.sigpro.2012.09.021.

[3] M. Scarpiniti, D. Comminiello, R. Parisi and A. Uncini, "Hammerstein uniform cubic spline adaptive filtering : learning and convergence properties," Signal Processing, 100, 112-123, 2014, doi:10.1016/j.sigpro.2014.01.019.

[4] M. Scarpiniti, D. Comminiello, R. Parisi and A. Uncini, "Novel cascade spline architectures for the identification of nonlinear systems," IEEE Transactions on Circuits and Systems I: Regular Papers, 62(7), 1825-1835, 2015, doi: 10.1109/TCSI.2015.2423791.

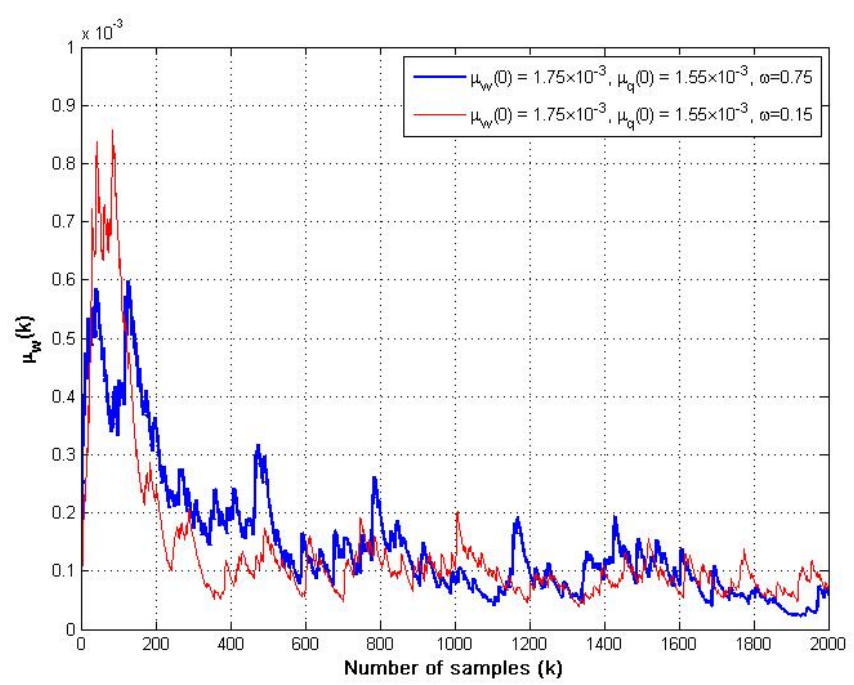

Figure 13: Trajectories of step-size $\mu_{w_{k}}$ of coefficient vector $\mathbf{w}_{k}$ using ECG input, when $\omega=0.15,0.75$ and $\mathrm{SNR}=25 \mathrm{~dB}$.

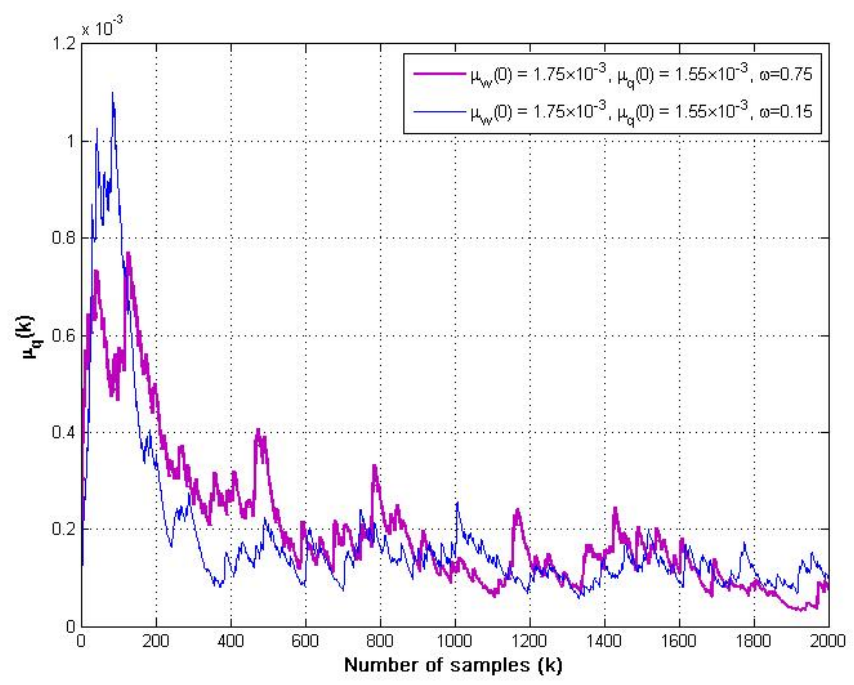

Figure 14: Trajectories of step-size $\mu_{q_{k}}$ of control points coefficient vector $\mathbf{q}_{i, k}$ using ECG input, when $\omega=0.15,0.75$ and $\mathrm{SNR}=25 \mathrm{~dB}$.

[5] C. Liu and Z. Zhang, "Set-membership normalised least M-estimate spline adaptive filtering algorithm in impulsive noise," Electronics Letters, 54(6), 393-395, 2018, doi:10.1049/el.2017.4434.

[6] C. Liu, C. Peng, X. Tang and X. Liu, "Two Variants of the IIR Spline Adaptive Filter for Combating Impulsive Noise", EURAZIP Journal on Advances in Signal Processing, 8, 1-15, 2019, doi:10.1186/s13634-019-0605-9.

[7] S. Prongnuch, S. Sitjongsataporn and T. Wiangtong, "Hammerstein Spline Adaptive Filtering based on normalized Least Mean Square Algorithm", in 2019 IEEE 2019 International Symposium on Intelligent Signal Processing and Communication Systems (ISPACS), 2019, doi:10.1109/ISPACS48206.2019.8986401.

[8] S. Prongnuch and S. Sitjongsataporn, "Stability and Steady-State Performance of Hammerstein Spline Adaptive Filter Based on Stochastic Gradient Algorithm ", International Journal of Intelligent Engineering and Systems, 13(3), 112-123, 2020, doi:10.22266/ijies2020.0630.11. 
T. Wiangtong et al. / Advances in Science, Technology and Engineering Systems Journal Vol. 6, No. 1, 577-586 (2021)

[9] C. Liu, Z. Zhang and X. Tang, "Sign Normalised Hammerstein Spline Adaptive Filtering Algorithm in an Impulsive Noise Environment", Neural Processing Letters, 50, 477-496, 2019, doi:10.1007/s11063-019-09996-6.

[10] C. Liu, Z. Zhang and X. Tang, "Steady-state Performance for the Sign Normalised algorithm based on Hammerstein Spline Adaptive Filtering", in 2019 IEEE International Conference on Control, Automation and Information Sciences (ICCAIS), 2019, doi:10.1109/ICCAIS46528.2019.9074547.

[11] C. Liu, Z. Zhang and X. Tang, "Sign Normalized Spline Adaptive Filtering Algorithms Against Impulsive Noise", Signal Processing, 148, 235-240, 2018, doi:10.1016/j.sigpro.2018.02.022.

[12] S. Zhang, J. Zhang, and H. Han, "Robust Shrinkage Normalized Sign Algorighm in an Impulsive Noise Environment", IEEE Transactions on Circuits and Systems, 64(1), 91-95, 2017, doi:10.1109/TCSII.2016.2546905.

[13] K. Xiong, S. Wang and B. Chen, "Robust Normalized Least Mean Absolute Third Algorithm", IEEE Access, 7, 10318-10330, 2019, doi:10.1109/ACCESS.2019.2891549.

[14] P. Wen and J. Zhang, "Robust Variable Step-size Sign Subband Adaptive Filter Algorithm against Impulsive Noise", Signal Processing, 139, 110-115, 2017, doi:10.1016/j.sigpro.2017.04.012.

[15] J. Kim, J. Choi, S.W. Nam, J. Chang, "Delayless Block IndividualWeighting-Factors Sign Subband Adaptive Filters With an Improved BandDependent Variable Step-Size",IEEE Access, 8, 185796 - 185803, 2020, doi:10.1109/ACCESS.2020.3029269.

[16] S. Zhang, J. Zhang and H. Han, "Robust Shrinkage Normalized Sign Algorithm in An Impulsive Noise Environment", IEEE Transactions on Circuits and Systems II: Express Briefs, 64(1), 91-95, 2017, doi: 10.1109/TCSII.2016.2546905.
[17] S. Guan and Z. Li, "Normalized Spline Adaptive Filtering Algorithm for Nonlinear System Identification", Neural Processing Letter, 46(2), 595-607, 2017, doi:10.1007/s11063-017-9606-6.

[18] S. Sitjongsataporn, W. Chimpat, "Adaptive Step-size Normalised Least Mean Square Algorithm for Spline Adaptive Filtering", in 2019 IEEE International Technical Conference on Circuits/Systems, Computers and Communications (ITC-CSCC), 544-547, 2019, doi:10.1109/ITC-CSCC.2019.8793383.

[19] A. Saengmuang and S. Sitjongsataporn, "Convergence and Stability Analysis of Spline Adaptive Filtering based on Adaptive Averaging Step-size Normalized Least Mean Square Algorithm ", International Journal of Intelligent Engineering and Systems, 13(2), 267-277, 2020, doi:10.22266/ijies2020.0430.26.

[20] S. Prongnuch and R.E. Valmoria, "Applied of Co-design in Reconfigurable System for Remote Image Noise Filtering via Ethernet Technology", in 2013 IEEE International Science, Social Science, Engineering and Energy Conference, 92-98, 2013.

[21] S. Sitjongsataporn, "Advanced Adaptive DMT Equalisation: Algorithms and Implementation”, LAP LAMBERT Academic Publishing, 2011.

[22] G.B. Moody and R.G. Mark, "A New Method for Detecting Atrial Fibrillation using R-R Intervals”, Computers in Cardiology, 10, 227-230, 1983.

[23] A. Goldberger, L. Amaral, L. Glass, J. Hausdorff, P.C. Ivanov, R. Mark, J.E. Mietus, G.B. Moody, C.K. Peng, and H.E. Stanley, "PhysioBank, PhysioToolkit, and PhysioNet: Components of a New Research Resource for Complex Physiologic Signals", Circulation [Online], 101(23), e215-e220, 2000, doi:10.1161/01.cir.101.23.e215.

[24] G. Moody and R. Mark, "MIT-BIH Atrial Fibrillation Database", 2000, [Online]. Available: https://physionet.org/content/afdb/1.0.0/. [Accessed Jan. 8, 2021], doi:10.13026/C2MW2D. 